\title{
Assessing the reporting and interpretation of non-significant results in the study of cognitive development: a systematic review
}

Edward W. Legg ${ }^{1 *}$, Benjamin G. Farrar ${ }^{2 *}$, Aleksandra Lazić ${ }^{3}$, Maleen Thiele ${ }^{4}$, Dora Kampis ${ }^{5}$, Nivedita Mani ${ }^{6}$, Kristina Sesar ${ }^{7}$, Eduard T. Klapwijk ${ }^{8,9}$, Laura Schlingloff ${ }^{10}$, Eva Reindl ${ }^{11}$, Kailing $\mathrm{Li}^{12}$, Gorana Birovljević ${ }^{1}$, Meg Attwood ${ }^{12}$, Denis Tatone ${ }^{10}$, Laras S. Yuniarto ${ }^{13}$, and Ljerka Ostojić $^{1}$

${ }^{1}$ Department of Psychology, Faculty of Humanities and Social Sciences, University of Rijeka

${ }^{2}$ Department of Psychology, University of Cambridge

${ }^{3}$ Department of Psychology, University of Belgrade

${ }^{4}$ Max Planck Institute for Evolutionary Anthropology, Department of Comparative Cultural Psychology

${ }^{5}$ Department of Psychology, University of Copenhagen

${ }^{6}$ Research Group Psychology of Language, Georg-August-University Göttingen

${ }^{7}$ Faculty of Humanities and Social Sciences, University of Mostar

${ }^{8}$ Erasmus School of Social and Behavioural Sciences, Erasmus University Rotterdam

${ }^{9}$ Brain and Development Research Center, Leiden University

${ }^{10}$ Cognitive Development Center, Department of Cognitive Science, Central European University

${ }^{11}$ School of Social Sciences, Birmingham City University

${ }^{12}$ School of Psychological Science, University of Bristol

${ }^{13}$ School of Psychology and Neuroscience, University of St Andrews

Author Note

*Edward W. Legg and Benjamin G. Farrar are co-first authors

Edward W. Legg https:/ / orcid.org/0000-0003-0353-7105

Benjamin G. Farrar https://orcid.org/0000-0001-8912-6133

Aleksandra Lazić https://orcid.org/0000-0002-0433-0483

Maleen Thiele https://orcid.org/0000-0002-1695-1850

Dora Kampis https://orcid.org/0000-0003-1678-3874

Nivedita Mani https://orcid.org/0000-0002-9843-4629

Kristina Sesar https:/ / orcid.org/0000-0003-2406-7114 
Non-Significant Results in Cognitive Development

Eduard T. Klapwijk https://orcid.org/0000-0002-8936-0365

Laura Schlingloff https://orcid.org/0000-0001-7512-2617

Eva Reindl https://orcid.org/0000-0003-1594-1367

Kailing Li https:/ /orcid.org/0000-0003-2563-2162

Gorana Birovljević https://orcid.org/0000-0001-6332-9223

Meg Attwood https://orcid.org/0000-0003-2576-1861

Denis Tatone https://orcid.org/0000-0001-6694-2656

Laras S. Yuniarto https://orcid.org/0000-0002-9221-6938

Ljerka Ostojić https:/ / orcid.org/0000-0002-8008-1773

Author Contribution Statement

Meg Attwood: methodology, writing - review and editing. Gorana Birovljević: methodology, writing - review and editing. Benjamin G. Farrar: conceptualization, methodology, writingreview and editing, supervision. Dora Kampis: methodology, writing - review and editing.

Eduard T. Klapwijk: methodology, writing - review and editing. Aleksandra Lazić: methodology, writing - review and editing. Edward W. Legg: conceptualization, methodology, writing - original draft preparation, supervision, project management. Kailing Li: methodology, writing - review and editing. Nivedita Mani: methodology, writing - review and editing. Ljerka Ostojić: conceptualization, writing - original draft preparation, supervision, project management. Eva Reindl: methodology, writing - review and editing. Laura Schlingloff: methodology, writing - review and editing. Kristina Sesar: methodology, writing - review and editing. Denis Tatone: methodology, writing - review and editing. Maleen Thiele: methodology, writing - review and editing. Laras S. Yuniarto: methodology, writing - review and editing.

email: ewlegg@gmail.com 


\begin{abstract}
Null Hypothesis Significance Testing is a statistical procedure widely used in cognitive development research. There is widespread concern that the results of this statistical procedure are misinterpreted and lead to unsubstantiated claims about studies' outcomes. Two particularly pertinent issues for research on cognitive development are: i) treating a non-significant result as evidence of no difference or no effect, and ii) treating a non-significant result in one group/condition and a significant result in another as evidence of a difference between groups/conditions. The current study focuses on quantifying the extent to which these two issues can be observed in the published literature on cognitive development. To this end, we will systematically search for empirical studies investigating cognitive development in 0-to-16-year-old children that have been published at two time points, namely in 1999 and 2019. For each of the two issues, we will extract information from 300 published articles, 150 per publication year.
\end{abstract}

Keywords: Cognitive Development; Meta-science; Null Hypothesis Significance Testing; systematic review; non-significant results; NHST; statistical comparisons 


\section{Introduction}

The study of human cognitive development investigates the changes in cognition that occur across the lifespan (Carey \& Markman, 1999; Oakley, 2004). Researchers investigate the presence and nature of a particular cognitive mechanism at different points in the lifespan and aim to develop theories to explain the observed changes (Goswami, 2008). In doing so, cognitive development researchers face a question that is common to all areas of empirical research: is it ever justifiable to make a generalisation from a limited number of observations (Hume 1739|2000)? Many of the statistical tests developmental scientists use in their day-to-day work have their origins in attempts to solve, or avoid, this philosophical challenge.

The statistical solutions to this challenge have provided an invaluable tool for scientists as they provide a formal means of relating observations with hypotheses. At the same time, it remains crucial to recognise that the results of different statistical approaches reflect different relationships between observations and hypotheses. One of the clearest differences is seen between Bayesian statistics and classic frequentist statistics. In Bayesian statistics, the data observed are used to update the probability of a hypothesis being true - which requires information about the probability of the hypothesis prior to the data being observed. In contrast, frequentist statistical tests provide information about the probability of data if a particular hypothesis is true. Like in the majority of fields in psychological sciences, it is this latter approach, known as "Null Hypothesis Significance Testing" (NHST; Gigerenzer, 2004; Perezgonzalez, 2015; Rozeboom, 1960), that is most commonly used by researchers in cognitive development.

Despite its common occurrence/use, it appears that many scientists mistakenly treat the outcomes of NHST as the probability of the hypothesis, which can lead to misinterpretations of these outcomes (Cohen, 1994; Gigerenzer, 1993; Goodman, 2008; Goodman, 1999; Hubbard \& Bayarri, 2003; Wasserstein \& Lazar, 2016), as reflected in the published literature across different research fields (Aczel et al., 2018; Fidler et al., 2006; Hoekstra et al., 2006; Nieuwenhuis et al., 2011). The exact origins of these misinterpretations and misconceptions are unclear, but a recurring criticism is that even textbooks employ shortcuts in explaining NHST (Gigerenzer, 2004, Rozeboom, 1960). Thus, it is critical to avoid shortcutting conceptual and philosophical aspects that form the basis of statistical approaches.

Consequently, here, we first provide some theoretical background about the different statistical approaches that are combined in NHST, namely Fisherian Significance Testing and NeymanPearson Hypothesis Testing. Next, building on this background, we focus on two issues that are especially pertinent for cognitive development research: i) treating a non-significant result as evidence of no difference or no effect, and ii) treating a non-significant result in one group/condition and a significant result in another group/condition as evidence of a difference between these groups/conditions. The aim of the current study is to assess the prevalence of these issues in the cognitive development literature at two time points.

\section{Frequentist statistical approaches}

Fisher developed his 'Significance Tests' as an alternative to Bayesian forms of inference because he was dissatisfied with the possibility that scientists setting different prior probabilities would end up with different posterior probabilities (Fisher, 1971). Fisher argued that his tests allowed generalisations to be made from sample to population without the need to estimate the prior probability (Hubbard \& Bayarri, 2003). To do this, Fisher proposed constructing a hypothesis that, if 'nullified', would aid judgements about the probability of an experimental manipulation having influenced the experimental results. Therefore, the test procedure involved 'constructing a 
hypothetical infinite population, of which the actual data are regarded as constituting a random sample' (Fisher, 1922, pg. 311). The probability of the observed or more extreme data being sampled from that hypothetical infinite population is represented by the p-value. Critically, for Fisher, the p-value represents a property of the sampled data itself ${ }^{1}$ and, thus, in terms of Fisher's aim of generalising from sample to population, he concluded that a small p-value had evidential value. Specifically, a low p-value indicated that "either an exceptionally rare chance has occurred or the theory [null hypothesis] is not true" (Fisher, 1956, p. 39). This disjunction is the inevitable result of the p-value reflecting a conditional probability based on an infinite population - the probability of the observed or more extreme data given the null hypothesis. Although the sampled data may be extremely improbable under the null hypothesis there is always a possibility that such rare events occur.

Neyman and Pearson built on Fisher's work but had a fundamentally different philosophical position on the role of induction and deduction for science and the nature of probability (Gigerenzer, 1993, 2004; Hubbard \& Bayarri, 2003; Neyman \& Pearson, 1933). Neyman and Pearson sought to rely solely on deduction to test statistical hypotheses, and in doing so they abandoned attempts to update beliefs about a hypothesis, instead focusing on ensuring efficient 'inductive behaviour' that would not be too wrong in the long run $^{2}$ (Lehmann, 2012; Mayo, 1985). Their 'Hypothesis Test' involves establishing two hypothetical populations, a null hypothesis ${ }^{3}$, and an alternative hypothesis with a different population mean difference to the null hypothesis. Critically, by setting an acceptable rate of false positives $(\alpha)$ over the long run, researchers can compute, for a particular statistical test and expected difference between null hypothesis and alternative hypothesis (i.e., effect size), the sample size necessary to achieve a pre-specified rate of false negatives $(\beta)$ in the long run). The long-term error rate laid out by $\alpha$ and $\beta$ reflect conditional probabilities - in the case of $\alpha$, this is the probability of making a mistake by rejecting the null hypothesis when it is true; in the case of $\beta$, this is the probability of making a mistake by rejecting the alternative hypothesis when it is true. Notably, unlike the Fisherian approach, $\alpha$ and $\beta$ are linked to the setup of a study and are properties of the test itself rather than the obtained data. As such they do not provide information about the probability of a single study but the probability of making incorrectly rejecting or accepting the null hypothesis across a series of tests.

\section{Null Hypothesis Significance Testing}

NHST combines elements of the Fisherian and Neyman-Pearson approaches. A crucial and consistent aspect of these views and subsequently NHST is that it tests the probability of data given a particular hypothesis. As explained earlier, researchers regularly and incorrectly treat the results of NHST as the probability of the hypothesis (Bakan, 1966; Falk \& Greenbaum, 1995; Gigerenzer, 2004). However, equating the probability of the data given the hypothesis with the probability of the hypothesis is problematic. The link between these two probabilities is provided by Bayes' theorem, which stipulates that the (posterior) probability of a hypothesis - if a particular observation has been made - is equal to the probability that the observation occurred when the hypothesis is true (a true positive observation) divided by the probability of the observation having occurred (total positive observations; see Etz \& Vandekerckhove, 2018 for an introduction to Bayesian theory). The probability of having made a true positive observation (in line with the null hypothesis) is calculated from the probability of the data given the null hypothesis - this corresponds to a $p$ value of a significant result in NHST - and the prior probability of the hypothesis - namely, how likely the null hypothesis is to be true. NHST provides the former but

\footnotetext{
${ }^{1}$ Fisher's view of what a probability was differed from the normal frequentist view of probabilities (for more information see: Lehmann, 1993, pg. 1245)

${ }^{2}$ Neyman and Pearson subscribed to a frequentist view of probability.
} 
not the latter. The total positive observations are calculated as the total number of true positive observations (as just described) plus the total number of false positive observations. The calculation of false positives involves multiplying the probability of obtaining the observed data if the null hypothesis is false with the prior probability of the hypothesis being false. The power of a statistical test influences the probability of obtaining a significant result if the effect is as big or greater than the one specified in the alternative hypothesis ${ }^{4}$. Consequently, the probability of detecting a significant difference when the null hypothesis is false with a low-powered test is less than in an equivalent study with higher power. The number of significant results that are reported when the null hypothesis is true will remain the same across the two studies. Thus, the proportion of significant results stemming from the null hypothesis being true will be expected to be higher in the study with the lower-powered statistical test.

The issue of power is important because low power tests a significant result gives little indication of whether the actual hypothesis in question is likely to be true. Estimates indicate that the power of the statistical tests used in neuroscience are low relative to the average effect size and this is likely to reduce the probability that a significant result reported in this literature reflects a true hypothesis (Button et al., 2013). Such issues may be relevant to cognitive development research, as highlighted by a recent study investigating looking time paradigms, which reported that most studies tend to use small sample sizes and as a consequence may be low-powered (Oakes, 2017).

For research on cognitive development the impact of the misinterpretation of NHST may be far reaching. One of the goals of the field is to investigate the nature of cognitive mechanisms at different time points. Here, misunderstandings about the meaning of $\mathrm{p}$-values may give rise to claims that cannot be justified using typical NHST procedures such as claims that 'an ability is absent' or that 'performance did not change with age'. A fundamental aspect of NHST is that a significant result leads to a different type of behaviour or change in attitude than a non-significant result. This asymmetry is inherent to NHST because researchers are testing whether the observed data are improbable under a particular hypothesis - finding that such data is expected under that hypothesis fails to provide information about whether it is expected under any other hypotheses. Fisher, who did not consider alternative hypotheses, proposed that non-significant results should be ignored and that experiments are only conducted to 'give the facts a chance of disproving the null hypothesis' (Fisher, 1971, p.16). Statistical tests that posit alternative hypotheses (i.e., those that make use of the Neyman-Pearson approach) and have high enough power for a specified effect size remain unable to 'accept' the null hypothesis but do indicate that if the two hypotheses were the only possibilities, then it would be better to act as if the null hypothesis is true. In line with this, Cohen (1988, pg. 16) has suggested that if a study with a non-significant result has high enough power, it is possible to reject that the difference in population means is at least as large as that implied under the alternative hypothesis. However, the rejection of the alternative hypothesis does not rule out that there is a difference between population means - there may be a difference, but it is likely to be smaller than the one specified under the alternative hypothesis. Despite explicit calls by the APA for researchers to stop 'accepting the null hypothesis' such claims remain prevalent in psychology (Aczel et al., 2018; Hoekstra et al., 2006; American Psychological Association, 2001). In addition, despite the existence of formal NHST techniques that can assess whether an effect size is smaller than one that would be of interest, such as equivalence tests, these are not widely used (Lakens, 2017; Wellek, 2002)

\footnotetext{
4 The power of a test is not necessarily the same as the probability of a true positive result because (depending on what one is testing) differences may exist that are smaller than the difference specified by the alternative hypothesis and the ability to detect these differences will be lower than the power of the test.
} 
A second and related common misinterpretation of $\mathrm{p}$-values that is highly relevant for cognitive development research concerns inferences regarding differences in effects between groups or conditions. There are concerns that researchers regularly infer such differences solely on the basis of one group showing a significant difference and another group not showing a significant difference (Gelman \& Stern, 2006; Goodman, 2008). However, such inferences need to be justified by a comparison between the groups. Significance relates to the probability of the observed or more extreme data if the population mean specified by the null hypothesis is true. As discussed previously, this does not mean that a non-significant result is more likely to be from the population specified in the null hypothesis than from any other population. For instance, it is possible that the means of the observed data that have led to a non-significant and significant difference are actually closer to each other than they are to the mean specified by the null hypothesis. An investigation into the rate at which the neuroscience literature justified such comparisons with statistical tests found approximately $50 \%$ of studies that made claims comparing the performance of two groups did so on the basis of one group having a significant difference and the other having a non-significant difference (Nieuwenhuis et al., 2011).

\section{Summary}

In summary, two important issues related to common misinterpretations of $p$-values within the NHST framework may be affecting the way that inferences about the development of cognitive abilities are made in the literature on cognitive development: i) 'acceptance' of the null hypothesis after a non-significant result, and ii) inference of differences between groups/conditions without direct and formal statistical assessment. Consequently, the aim of the current project is to explore the way that statistical results are reported and interpreted in the literature on cognitive development in regard to these two issues. To do so, we will conduct a systematic review of published empirical studies on cognitive development in 0- to 16-year-old children and categorise: i) how non-significant results are reported and interpreted, and ii) whether or not differences in effects between groups/conditions are inferred on the basis of a direct statistical test. Furthermore, although statisticians have been highlighting problems with the way in which NHST is used for a long time (Bakan, 1966; Rozeboom, 1960), the last two decades have brought an increased awareness of the issues around statistical tests and this has potentially altered practices in some fields (Christensen et al., 2019; Fidler et al., 2004; Ioannidis, 2005; Sassenberg \& Ditrich, 2019). Within psychology, many researchers now perceive that failures to replicate previous studies are, at least in part, due to the use of low powered statistical tests and misconceptions about how likely a study with a significant result is to replicate (Goodman, 1992; Simonsohn, 2015). Within cognitive development research, there have been recent movements to address some of these issues by fostering multi-lab collaborations (ManyBabies, n.d.; ManyBabies Consortium, 2020). Thus, we will describe how statistical tests are reported and interpreted in regard to the two issues at two time points, namely once before the APA issued explicit guidance on reporting null results (1999) and two decades later (2019). 


\section{Methods}

\section{Sampling}

To identify key words for a subsequent literature search, all members of the research team were asked to provide words or terms that they believe capture the core research focus of cognitive development research. 245 terms were provided by the team resulting in 179 search terms (both lists are presented in the supplemental material). The literature search will follow PRISMA guidelines (Prisma checklist is included in the Supplemental Material, PRISMA Diagram: Figure 1; Page et al., 2021) and will be conducted using Web of Science with the search history included in the supplemental material.

Next, we will search for articles that include the terms from Table 1 in their title, abstract or keywords. In addition to the keywords listed by authors in their publication, for each indexed article, Web of Science generates a list of keywords based on the article's title (Web of Sciences groups a search of these features as 'Topic'). Thus, the output of the search of the terms in keywords will be the result of both sets of keywords.

Two separate searches will be conducted: one that includes papers published in 1999 and one that includes papers published in 2019. These will be ordered by date (oldest first) using the Web of Science system and will then be exported for the screening stage.

\section{Screening}

Screening will be conducted on all articles that are outputted from the Web of Science search to ensure that they fit the inclusion criteria in a two-step process, as detailed below. Step 1 (conducted by one screener through a combination of manual and automated methods; EWL) will ensure that the articles fit the general inclusion criteria, and Step 2 (conducted by two screeners separately and manually; EWL and LO) will ensure that the articles fit the specific inclusion criteria for the two research questions (see Figure 1 for a diagrammatic representation of the screening strategy).

The final target outcome of the screening process is 150 articles for each time period for each of the two research questions ${ }^{5}$. To achieve this outcome, the whole screening process will be conducted in the following manner. The first screener (EWL) will, for each time period (1999 and 2019), work backward through the list of articles outputted by Web of Science, starting with the article with the latest publishing date. For each article, the first screener (EWL) will screen for the general inclusion criteria (Step 1), and if all are satisfied, they will immediately proceed to code the article for the specific inclusion criteria of both research questions (Step 2). They will continue coding articles on the outputted list in this manner until they have identified 300 articles that satisfy both the general and the specific inclusion criteria for each research questions, 150 for each time period.

Subsequently, a list of all articles that meet the general inclusion criteria will be sent to the second screener (LO) who will independently code for the specific inclusion criteria (i.e., the second screener will be blind to the first screener's coding for the specific inclusion criteria) until they identify 150 articles fitting the criteria for each research question per publication year. Any discrepancies between the two coders' selections for each research question will be resolved

\footnotetext{
${ }^{5}$ If too few papers from the selected years meet the inclusion criteria for each research question, then the searches will be extended to include the years 1998 and 2018, respectively.
} 
through discussion - where this leads to more than 150 articles being agreed on per year for each research question, the 150 articles with the latest publication dates will be included. Where this leads to fewer than 150 articles being selected per year, the screening process will be repeated for an additional number of articles to make up the shortfall.

\section{Step 1: General inclusion criteria}

Articles will be screened to ensure they fit the following general inclusion criteria: i) the article is not a duplicate of another article, ii) the output is an original research article (i.e., the article reports an empirical piece of research) published in a peer-reviewed journal (i.e., not conference proceedings and similar), iii) the whole article is accessible to at least one member of the research team, iv) the article is published in English ${ }^{6}$, v) the article is on cognitive development, and vi) the article tests a sample of typically developing children aged $0-16$ years.

The screening process will be conducted using both automated and manual procedures. Table 1 presents the decision process, with automated procedures being run first using the data exported from Web of Science (see Appendix for the R code). For the manual procedure, the inclusion criteria i), ii), and v) will be assessed from the abstract. Criteria iii) and iv) will be assessed by searching for an accessible version of the article on the internet or through institutional subscriptions first of the screener and then the other members of the research team. For this purpose, the screener will post a request for the article to the research team using their Slack channel and will wait three days for a reply. Where the article is not accessible through these means, a request for the author's version of the manuscript will be sent to the corresponding author. If the corresponding author does not respond with a version of the manuscript within seven working days, then the article will be excluded. Criterion vi) will in most cases be coded from the article's abstract, however in cases where information about demographics (age and development) is not provided in the abstract, the Methods section will be consulted. Articles will be screened in the order in which the criteria are outlined here, such that if an article does not satisfy one criterion, it will not be assessed on subsequent one(s). Only articles that satisfy all general inclusion criteria will be screened for the specific inclusion criteria.

Table 2. Screening 1. inclusion criteria including description of automated and manual procedures. The $\mathrm{R}$ code for the automated screening is reported in the Appendix.

\begin{tabular}{cccc} 
Label & $\begin{array}{c}\text { Inclusion } \\
\text { Criteria }\end{array}$ & Automated Procedure & Manual Procedure \\
\hline i & Not a duplicate & $\begin{array}{c}\text { Flag articles that have matching } \\
\text { 'Title' or 'DOI'. }\end{array}$ & $\begin{array}{c}\text { Check whether the flagged articles } \\
\text { are duplicates }\end{array}$ \\
\hline ii & $\begin{array}{c}\text { Original } \\
\text { research article }\end{array}$ & $\begin{array}{c}\text { Select documents labelled as } \\
\text { "Article" }\end{array}$ & $\begin{array}{c}\text { Only select articles that include } \\
\text { original research: for example, no } \\
\text { review articles or conference } \\
\text { proceedings }\end{array}$ \\
\hline iii & $\begin{array}{c}\text { Accessible to } \\
\text { research team }\end{array}$ & NA & $\begin{array}{c}\text { Web-search/institutional acess by } \\
\text { screener; co-author instituational } \\
\text { access; email to authors }\end{array}$ \\
\hline iv & $\begin{array}{c}\text { Published in } \\
\text { English }\end{array}$ & Select articles labelled as 'English' & $\begin{array}{c}\text { Only include articles that have } \\
\text { abstract, methods, results and } \\
\text { discussion written in English }\end{array}$ \\
\hline
\end{tabular}

\footnotetext{
${ }^{6}$ Only including English publications restricts this study's generalisability as reporting practices may differ for publications in other languages. For example, one study found differences in effect size estimates between ecological meta-analyses that included publications only in English and those that included both Japanese and English language articles (Konno et al., 2020).
} 


\begin{tabular}{|c|c|c|c|}
\hline $\mathbf{v}$ & $\begin{array}{l}\text { On cognitive } \\
\text { development }\end{array}$ & $\begin{array}{c}\text { Select articles in the research areas } \\
\text { 'behavioral sciences' and } \\
\text { 'psychology' }\end{array}$ & $\begin{array}{l}\text { Only select articles on cognitive } \\
\text { development: are these articles on } \\
\text { the development of cognitive } \\
\text { processes? - use search criteria } \\
\text { from Table } 1 \text { to help define } \\
\text { 'cognitive processes' }\end{array}$ \\
\hline vi & $\begin{array}{l}\text { Typically } \\
\text { developing } 0- \\
\text { 16yr olds }\end{array}$ & NA & $\begin{array}{l}\text { Only include articles that have at } \\
\text { least one group of typically } \\
\text { developing children aged } 0-16 y r s ; \\
\text { screener will note down mean age } \\
\text { of youngest and oldest groups }\end{array}$ \\
\hline
\end{tabular}

\section{Step 2: Specific inclusion criteria}

Screening for specific inclusion criteria will be conducted separately for each research question. For both research questions screening step 2 relates to statements about the analyses and results of a study reported in the abstract. Any statement selected for screening step 2 need to be about the relevant populations as defined in screening step 1.

For Research Question 1 (reporting and interpreting of negative results), the inclusion criterion refers to whether the article contains a 'negative statement' that is based on the use of NHST. Our definition of a negative statement will follow the definition used in Aczel et al. (2018), namely any statement about the absence of an effect or a non-significant result. To assess this inclusion criterion, the abstract will be screened for a 'negative statement' and if there is one, then the Results section will be screened to assess whether the statistical analysis supporting this negative statement was conducted using NHST.

For Research Question 2 (comparison of significant and non-significant results), the inclusion criteria refer to: i) whether an article contains a statement implying a difference regarding the same dependent variable(s) between at least two conditions or groups, where it would be possible, with the data obtained from the study, for the authors to have made the statement - albeit without justification - by contrasting a significant and non-significant result, and ii) whether the statistical test(s) the authors based their statement on were solely NHST. For simplicity, we will refer to this as an 'interaction claim'.

The typical example of a comparison to be included involves a study with at least 2 independent variables both with at least 2 levels. For example, a $2 \times 2$ design where the dependent variable is the number of items remembered, one independent variable is age with 2 levels - 3 and 5 years - and the second independent variable is cognitive load with 2 levels. Here, it would be possible to compare whether the increase in cognitive load affects the two age groups' performance levels differently using an ANOVA and it would also be possible for the comparison to be made - in an unjustified manner - when for example, a t-test run on 5-year olds revealed a significant effect of cognitive load and a t-test run on the 3-year-olds did not detect a significant difference.

We will also include studies that do not involve a minimum of a $2 \times 2$ design but where the two conditions or groups implicated in the statement are tested against chance or any other expected value and where this comparison is implied in the abstract. For example, a study that ran a binomial test to compare 3-year-olds' performance to chance and another binomial test comparing 5-yearolds' performance to chance would be included even though it does not have a $2 \times 2$ design. 
If the article meets these criteria to be included in Research Question 2, then the screener will immediately proceed to coding.

Next, all articles that successfully satisfy the general inclusion criteria will be forwarded to the second screener, who will have access to the first screener's coding of the general inclusion criteria but not the specific inclusion criteria (i.e., the second screener will be blind to the first screener's coding of the specific inclusion criteria). After the second screener has finished the screening, the two screeners' coding for the specific inclusion criteria will be compared. Any disagreement between the two screeners will be discussed and a consensus reached on whether the article should be included or excluded for further coding. After having resolved any disagreements, if there are fewer than 150 papers for each time period for each research question, further papers will be screened. For this, the first screener will again conduct the screening for general inclusion criteria and specific inclusion criteria and will forward to the second screener all articles satisfying all general inclusion criteria. Depending on how many articles are outstanding, the screeners will decide on the number of coded articles in this process, i.e., when the first screener will forward the articles to be screened to the second screener.

At the end of the Steps 1 and 2, a list of articles that satisfy the inclusion criteria for each research question will be compiled for the main coding. An article that meets the inclusion criteria for one research question will not necessarily meet the inclusion criteria for the other research question, however, if an article satisfies the specific inclusion criteria for both research questions, it will be represented on both lists.

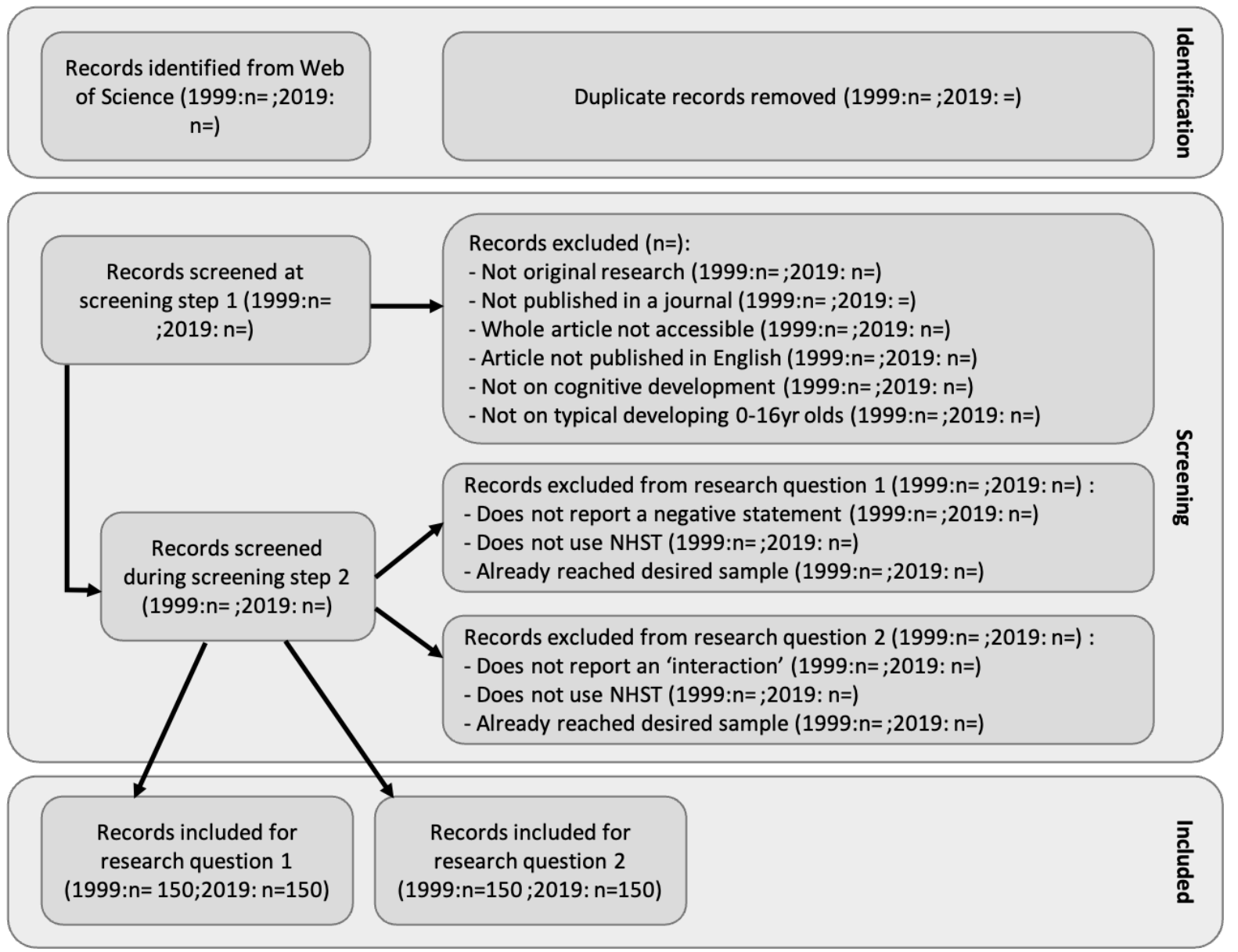

Figure 1. Flow chart of the screening procedure and criterion for exclusion. Note that the numbers at each step will be filled in for the stage 2 report. 


\section{Coding}

The coding for Research Question 1 will be conducted by each member of the research team. Each coder will be randomly assigned 20 articles. Each coder will receive a CSV file including basic information about the article including title, authors, journal, and DOI. The coding primarily consists of identifying particular information in an article (in which case the coder is asked to copy and paste the text from the article into the CSV file) and in categorising the identified text according to a pre-specified coding scheme.

The coding for Research Question 2 will be conducted at the same time as Step 2 by EWL.

\section{Training}

For research question 1, each coder will be trained to use the coding scheme whereby each coder will code the same 6 papers. The papers selected - 3 from the year 2000 and 3 from 2020 - will not be part of the final sample. All will include a negative statement. The coders will receive feedback about whether their classifications fit with the coding scheme and the reasons for any discrepancies. At this point, we will discuss whether any minor changes to the coding instructions are required.

For research question 2, EWL and LO will undergo training on a further 6 papers - 3 from the year 2000 and 3 from 2020. They will provide feedback on each other's responses and make any minor changes to the coding instructions that may be required.

\section{Research Question 1}

To investigate how negative results within an NHST framework are reported and interpreted in the published literature on cognitive development, we will identify and categorise statements that are made in the abstract, results section, and discussion that relate to the main negative claim of the paper. In addition, for the abstract, we will distinguish between a statement about the study's results and a statement that involves a generalized substantive claim. Previous studies have made similar distinctions based on whether authors make a statement about a population or a sample (Aczel et al., 2018). However, we feel that such a distinction could lead to confusion because the Fisherian and Neyman-Pearson approaches have different views about the nature of the statistical population that is being tested (Rubin, 2020). To avoid these concerns, we chose to distinguish between: i) statements about the 'results of the current study' that do not involve any generalisations beyond the particulars of the study (such as the population tested, experimental manipulation used or any other aspect of the study), and ii) statements that use the statistical results to make general substantive claims beyond the particular features of the experiment (e.g., to the population that the sample was drawn from, and/or the concept or theoretical construct that the experiment manipulation was thought to measure).

An overview of the information that will be coded from each section of the article is presented in the list below and full details of the instructions presented to the coders who will make these decisions are presented in the Coding Scheme in the Appendix.

\section{Abstract}

$$
\text { 1.1 Identifying the negative statement(s) }
$$


Coders are asked to copy and paste the sentence(s), from the abstract, containing the main negative statement. If applicable, coders are asked to do this in one column for a statement that is about the current study's results, and in a second column for a generalized substantive claim. If an abstract contains more than one negative statement, coders are asked to select which one they believe to be the main one, i.e., which one they believe to be closer to the main claim of the article. Coders may take into account the title of the article if it helps them decide. If two statements appear to have equal importance, coders are asked to select the first one reported in the abstract.

\subsection{Categorising the 'results of current study' statement}

If applicable, coders are asked to categorise the statement that directly reports the results in the abstract according to one of the following categories:

a) 'Non-significant or literally correct': reports that there was no significant difference between two conditions/groups or to theoretically expected values, or words to that effect, or reports of a correct directional statement, or reports where the actual means were exactly the same.

b) 'No Effect': statements that there was not a difference within the sample, when in fact there was - it was just not significant in the analysis.

c) 'Similar or small effect size': statements that suggest that the samples were similar without stating either that they were exactly the same, or that there was a significant difference between them.

d) 'Cannot Tell': statements that are extremely difficult to interpret or that do not fit any of the other categories.

\subsection{Categorising the 'generalized substantive claim'}

If applicable, coders are asked to categorise the most generalized substantive claim that directly relates to the study in the abstract according to one of the following categories:

a) 'Justified': statement commenting on statistical power, use of equivalence tests or otherwise a justification as to why a non-significant result suggests that there is no theoretically important difference, or that the study provides no strong evidence of a difference.

b) 'No Effect': interpretations of a non-significant result as showing that $\mathrm{X}$ and $\mathrm{Y}$ do not differ.

c) 'Caveated': interpretations of a non-significant result that describe the result as suggesting/indicating that $\mathrm{X}$ and $\mathrm{Y}$ do not differ.

\section{Methods}

\subsection{Identifying any a priori power analysis}

Coders are asked to identify whether the authors conducted an a priori power analysis and if so, to copy and paste the relevant sentence(s).

\section{Results}

\subsection{Identifying the statistical analysis supporting the main negative statement}

Coders are asked to copy and paste the sentence(s), from the results section (or results and discussion section where applicable), that present the statistical result supporting the main negative statement.

\subsection{Categorising the reporting of the statistical analysis}

Coders are asked to categorise the statement that directly reports the results of the statistical analysis using the same four categories used to categorise the 'results of the current study' from the abstract. These are presented earlier in this list at point 1.3. 


\section{Discussion}

\subsection{Identifying discussion of main negative claim}

Coders are asked to identify whether the authors discuss the main negative claim in the discussion section. If yes, they are asked to copy and paste the sentence(s) that they feel are the most important and relevant points the authors are making regarding the interpretation of the negative result.

\subsection{Identifying discussions of statistical power}

Coders are also asked to copy and paste in one column any sentence(s) relating to discussion of statistical power.

\subsection{Identifying discussion of sample size}

Coders are also asked to copy and paste in one column any sentence(s) relating to discussion of sample size. In some cases, this sentence will be the same as the sentence relating to statistical power.

\section{Research Question 2}

To investigate whether interaction statements are supported by formal statistical analyses within an NHST framework, we will code the following aspects of each article:

\section{Abstract}

1.1 Identifying the main 'interaction claim'. Coders are asked to copy and paste the sentence(s) that refer to a comparison of an effect between two conditions or groups. If applicable, coders are asked to do this for both a statement that directly reports the results and a statement that makes the most generalised substantive claim that directly relates to the study. If an abstract contains more than one 'interaction claim', coders are asked to select which one they believe to be the main one, i.e., which one they believe to be closer to the main claim of the article. Coders may take into account the title of the article if it helps them decide. If two statements appear to have equal importance, coders are asked to select the first one reported in the abstract.

\section{Results}

2.1 Identifying the statistical analysis surrounding the main 'interaction claim'. Coders are asked to copy and paste the sentence(s), from the results section (or result and discussion section where applicable), that present the statistical result surrounding the main interaction statement.

2.2 Categorising the statistical analysis surrounding the main 'interaction claim'. Coders are asked to categorise the statistical analysis according to one of the following categories:

a) 'Directly tested': the difference was formally tested, for example through an interaction term or through a different but direct statistical comparison between groups/conditions.

b) 'Not directly tested': the difference between groups/conditions was not formally tested but was interpreted based on a positive result in one group/condition and a negative result in the other group/condition.

\section{Reliability and Quality Control}

\section{Research Question 1}


Twenty percent of all articles will be coded by EWL who will be blind to both the statements selected by the other coders and their categorisations - the articles to be double-coded will be selected at random. EWL will first extract the statements. Following this, the coder will be given the statements selected by the first coder from a random $20 \%$ of articles and asked to categorise them. Inter-rater agreement for both steps of the double-coding procedure will be calculated by overall percentage agreement. For the second step it is important to show not only if there is overall disagreement but for each category what direction the disagreement was in (e.g., when the original coder coded a "generalised substantive claim" as "justified", the percentage of these claims the second coder rated as "justified", "no effect" and "caveated).

In addition, all articles will undergo a non-blind quality control procedure, which primarily aims at identifying and removing errors from the dataset. Second coders (each member of the research team excluding EWL) will review the statements identified and categorised by the original coders. For this, each second coder will receive a file with the data extracted by one of the original coders. The second coder will verify that the statements extracted by the first coder are consistent with the coding guidelines, e.g., that negative statements are truly negative, and that the extracted statistical analysis corresponds to this negative statement. If the second coder identifies a mistake, they will classify this as a major disagreement, and if they disagree with the original coding but are uncertain regarding their judgement, they will classify this as a minor disagreement. EWL will review all disagreements and make a final decision, returning to the original article if necessary.

The main purpose of the quality control procedure is to minimise mistakes. However, the procedure will also highlight where the coders had most difficulties in agreeing about the claims and categorisations. The reliability testing procedure will provide an additional means of quantifying agreement for each variable. Because the current project aims to investigate how different results are reported and interpreted, it is not the aim to define the coding variables so narrowly that high inter-rater agreement is guaranteed. Rather, we are interested in how experts in a research field interpret the claims and reports of statistical analyses in the published literature. Thus, any variables and situations for which there may exist a large variation in how they are categorised by the different coders can be meaningful, as they may highlight the ambiguity in the language used to report results. To this end, we will report the percentage of major and minor agreements between coders for each category as well as the percentage of each decision that was altered as a result of this quality control procedure.

\section{Research Question 2}

Twenty percent of all selected articles will be coded by one member of the research team (to be selected at a later date) who will be blind to the decision of the first coder. They will first be asked to categorise the main 'interaction claim' reported in the paper. Following this, the blind coder will be given the 'interaction claims' selected by the first coder and asked to categorise these. Interrater agreement for both steps of the double-coding procedure will be measured by overall percentage agreement. In addition, for the second step we will report the direction of the disagreements between coders (e.g. when the first coder coded a claim as being based on a "direct test" the percentage of these claims the second coder coded as being a "direct test" or "not directly tested".)

In addition, all articles will undergo a non-blind quality control procedure, which primarily aims at identifying and removing errors from the dataset. A second coder will review the statements identified and categorised by the original coders. For this, the second coder (a single member of the research team) will receive a file with the data extracted by the first coder. The second coder will verify that the statements extracted by the first coder are consistent with the coding guidelines 
for research question 2 and whether they agree with the first coder's categorisation of the statistical test. If the second coder identifies a mistake, they will classify this as a major disagreement, and if they disagree with the original coding but are uncertain regarding their judgement, they will classify this as a minor disagreement. A third person (to be selected at a later time point) will review all disagreements and make a final decision, returning to the original article if necessary. We will report the percentage of major and minor agreements between coders for each category as well as the percentage of each decision that was altered as a result of this quality control procedure.

\section{Analysis}

All analyses will be descriptive. For each research question and time period, we will present the percentage of statements per coded category. To illustrate the types of claims in each category, we will provide examples in tables. In addition, all data and code used for calculating the reported descriptive statistics including those used for inter-rater agreement will be made openly accessible. 


\section{References}

Aczel, B., Palfi, B., Szollosi, A., Kovacs, M., Szaszi, B., Szecsi, P., Zrubka, M., Gronau, Q. F., van den Bergh, D., \& Wagenmakers, E.-J. (2018). Quantifying Support for the Null Hypothesis in Psychology: An Empirical Investigation. Advances in Methods and Practices in Psychological Science, 1(3), 357-366. https://doi.org/10.1177/2515245918773742

Bakan, D. (1966). The test of significance in psychological research. Psychological Bulletin, 66(6), 423-437. https://doi.org/10.1037/h0020412

Button, K. S., Ioannidis, J. P. A., Mokrysz, C., Nosek, B. A., Flint, J., Robinson, E. S. J., \& Munafò, M. R. (2013). Power failure: Why small sample size undermines the reliability of neuroscience. Nature Reviews Neuroscience, 14(5), 365-376.

https://doi.org/10.1038/nrn3475

Carey, S., \& Markman, E. M. (1999). Cognitive Development. In Cognitive Science (pp. 201-254). Elsevier. https://doi.org/10.1016/B978-012601730-4/50007-X

Christensen, G., Wang, Z., Paluck, E. L., Swanson, N., Birke, D. J., Miguel, E., \& Littman, R. (2019). Open Science Practices are on the Rise: The State of Social Science (3S) Survey. MetaArXiv. https://doi.org/10.31222/osf.io/5rksu

Cohen, J. (1988). Statistical power analysis for the behavioral sciences (2nd ed). L. Erlbaum Associates.

Cohen, J. (1994). The earth is round ( $\mathrm{p}<$.05). American Psychologist, 49(12), 997-1003. https://doi.org/10.1037/0003-066X.49.12.997

Etz, A., \& Vandekerckhove, J. (2018). Introduction to Bayesian Inference for Psychology. Psychonomic Bulletin \& Review, 25(1), 5-34. https:/ / doi.org/10.3758/s13423-017-1262-3

Falk, R., \& Greenbaum, C. W. (1995). Significance Tests Die Hard: The Amazing Persistence of a Probabilistic Misconception. Theory \& Psychology, 5(1), 75-98. https://doi.org/10.1177/0959354395051004 
Fidler, F., Burgman, M. A., Cumming, G., Buttrose, R., \& Thomason, N. (2006). Impact of Criticism of Null-Hypothesis Significance Testing on Statistical Reporting Practices in Conservation Biology. Conservation Biology, 20(5), 1539-1544.

https://doi.org/10.1111/j.1523-1739.2006.00525.x

Fidler, F., Geoff, C., Mark, B., \& Neil, T. (2004). Statistical reform in medicine, psychology and ecology. The Journal of Socio-Economics, 33(5), 615-630. https://doi.org/10.1016/j.socec.2004.09.035

Fisher, R. A. (1922). On the Mathematical Foundations of Theoretical Statistics. Philosophical Transactions of the Royal Society of London. Series A, Containing Papers of a Mathematical or Physical Character, 222, 309-368.

Fisher, R. A. (1956). Statistical Methods and Scientific Inference. Edinburgh, Oliver and Boyd.

Fisher, R. A. (1971). The Design of Experiments (9th edition). Macmillan Pub Co.

Gelman, A., \& Stern, H. (2006). The Difference Between "Significant" and "Not Significant" is not Itself Statistically Significant. The American Statistician, 60(4), 328-331. https://doi.org/10.1198/000313006X152649

Gigerenzer, G. (1993). The superego, the ego, and the id in statistical reasoning. In $A$ bandbook for data analysis in the behavioral sciences: Methodological issues (pp. 311-339). Lawrence Erlbaum Associates, Inc.

Gigerenzer, G. (2004). Mindless statistics. The Journal of Socio-Economics, 33(5), 587-606. https://doi.org/10.1016/j.socec.2004.09.033

Goodman, S. (2008). A dirty dozen: Twelve p-value misconceptions. Seminars in Hematology, 45(3), 135-140. https://doi.org/10.1053/j.seminhematol.2008.04.003

Goodman, S. N. (1992). A comment on replication, p-values and evidence. Statistics in Medicine, 11(7), 875-879. https://doi.org/10.1002/sim.4780110705 
Goodman, S. N. (1999). Toward Evidence-Based Medical Statistics. 1: The P Value Fallacy. Annals of Internal Medicine, 130(12), 995. https:/ / doi.org/10.7326/0003-4819-130-12199906150-00008

Goswami, U. (2008). Cognitive Development: The Learning Brain (1st edition). Psychology Press.

Hoekstra, R., Finch, S., Kiers, H. A. L., \& Johnson, A. (2006). Probability as certainty:

Dichotomous thinking and the misuse ofp values. Psychonomic Bulletin \& Review, 13(6), 1033-1037. https://doi.org/10.3758/BF03213921

Hubbard, R., \& Bayarri, M. J. (2003). Confusion Over Measures of Evidence (p’s) Versus Errors ( $\alpha$ 's) in Classical Statistical Testing. The American Statistician, 57(3), 171-178.

https://doi.org/10.1198/0003130031856

Hume, D., Norton, D. F., \& Norton, M. J. (Eds.). (2000). A Treatise of Human Nature: Being an Attempt to Introduce the Experimental Method of Reasoning into Moral Subjects. Oxford University Press.

Ioannidis, J. P. A. (2005). Why Most Published Research Findings Are False. PLOS Medicine, 2(8), e124. https://doi.org/10.1371/journal.pmed.0020124

Konno, K., Akasaka, M., Koshida, C., Katayama, N., Osada, N., Spake, R., \& Amano, T. (2020). Ignoring non-English-language studies may bias ecological meta-analyses. Ecology and Evolution, 10(13), 6373-6384. https://doi.org/10.1002/ece3.6368

Lakens, D. (2017). Equivalence Tests. Social Psychological and Personality Science, 8(4), 355-362. https://doi.org/10.1177/1948550617697177

Lehmann, E. L. (1993). The Fisher, Neyman-Pearson Theories of Testing Hypotheses: One Theory or Two? Journal of the American Statistical Association, 88(424), 1242-1249. https://doi.org/10.1080/01621459.1993.10476404

Lehmann, E. L. (2012). Neyman's Statistical Philosophy. In J. Rojo (Ed.), Selected Works of E. L. Lehmann (pp. 1067-1073). Springer US. https://doi.org/10.1007/978-1-4614-1412-4_90 ManyBabies. (n.d.). Retrieved September 10, 2021, from https://manybabies.github.io/ 
Non-Significant Results in Cognitive Development

ManyBabies Consortium. (2020). Quantifying Sources of Variability in Infancy Research Using the Infant-Directed-Speech Preference. Advances in Methods and Practices in Psychological Science, 3(1), 24-52. https://doi.org/10.1177/2515245919900809

Mayo, D. G. (1985). Behavioristic, Evidentialist, and Learning Models of Statistical Testing. Philosophy of Science, 52(4), 493-516.

Neyman, J., \& Pearson, E. S. (1933). On the Problem of the Most Efficient Tests of Statistical Hypotheses. Philosophical Transactions of the Royal Society of London. Series A, Containing Papers of a Mathematical or Physical Character, 231, 289-337.

Nieuwenhuis, S., Forstmann, B. U., \& Wagenmakers, E.-J. (2011). Erroneous analyses of interactions in neuroscience: A problem of significance. Nature Neuroscience, 14(9), 11051107. https://doi.org/10.1038/nn.2886

Oakes, L. M. (2017). Sample Size, Statistical Power, and False Conclusions in Infant LookingTime Research. Infancy, 22(4), 436-469. https://doi.org/10.1111/infa.12186

Oakley, L. (2004). Cognitive Development. Routledge. https://doi.org/10.4324/9780203482834

Page, M. J., McKenzie, J. E., Bossuyt, P. M., Boutron, I., Hoffmann, T. C., Mulrow, C. D., Shamseer, L., Tetzlaff, J. M., Akl, E. A., Brennan, S. E., Chou, R., Glanville, J., Grimshaw, J. M., Hróbjartsson, A., Lalu, M. M., Li, T., Loder, E. W., Mayo-Wilson, E., McDonald, S., ... Moher, D. (2021). The PRISMA 2020 statement: An updated guideline for reporting systematic reviews. BMJ, 372, n71. https://doi.org/10.1136/bmj.n71

Perezgonzalez, J. D. (2015). Fisher, Neyman-Pearson or NHST? A tutorial for teaching data testing. Frontiers in Psychology, 6. https://doi.org/10.3389/fpsyg.2015.00223

Rozeboom, W. W. (1960). The fallacy of the null-hypothesis significance test. Psychological Bulletin, 57(5), 416-428. https://doi.org/10.1037/h0042040

Rubin, M. (2020). "Repeated sampling from the same population?" A critique of Neyman and Pearson's responses to Fisher. European Journal for Philosophy of Science, 10(3), 42. https://doi.org/10.1007/s13194-020-00309-6 
Non-Significant Results in Cognitive Development

Sassenberg, K., \& Ditrich, L. (2019). Research in Social Psychology Changed Between 2011 and 2016: Larger Sample Sizes, More Self-Report Measures, and More Online Studies. Advances in Methods and Practices in Psychological Science, 2(2), 107-114. https://doi.org/10.1177/2515245919838781

Simonsohn, U. (2015). Small Telescopes: Detectability and the Evaluation of Replication Results. Psychological Science, 26(5), 559-569. https://doi.org/10.1177/0956797614567341

Society, A. P. (2001). Publication Manual of the American Psychological Association (5th edition). Amer Psychological Assn.

Wasserstein, R. L., \& Lazar, N. A. (2016). The ASA Statement on p-Values: Context, Process, and Purpose. The American Statistician, 70(2), 129-133.

https://doi.org/10.1080/00031305.2016.1154108

Wellek, S. (2002). Testing Statistical Hypotheses of Equivalence. Chapman and Hall/CRC. https://doi.org/10.1201/9781420035964 